\title{
ON ELECTROSTATIC AHARONOV-BOHM EFFECT IN SOLIDS
}

\author{
T. Figielski, T. Wosiński, A. Morawski and A. Mąkosa \\ Institute of Physics, Polish Academy of Sciences \\ Al. Lotników 32/46, 02-668 Warszawa, Poland
}

We analyse conditions for an appearance of the electrostatic AharonovBohm interference in two systems: a single-channel quantum-wire loop and an open ballistic quantum dot. We show that in the first system the effect will be destroyed by charge fluctuations, which probably is the reason why it has not been clearly observed, while in the second system the effect is still open for exploration.

PACS numbers: 03.65.Bz, 72.15.Gd

We show first that ballistic electron in a single-channel quantum wire acquires a quantum-mechanical phase as large as $\pi / 2$ when electric charge equal to the elementary charge is accumulated on a condenser formed by a gate electrode coupled capacitively to the wire. Therefore, the electrostatic Aharonov-Bohm (eAB) effect predicted in mesoscopic conducting loops [1] will be readily destroyed by charge fluctilations. In fact, the electrostatic $\mathrm{AB}$ effect has never been directly demonstrated in solids except when it was observed in a combination with the magnetostatic effect $[2,3]$.

Let us take into account a loop made of a single-channel.quantum wire where a bias voltage is applied between the wire and a gate electrode coupled capacitively to one branch of the loop (Fig. 1). An electron at the Fermi level, travelling ballistically over the wire, acquires a phase $\varphi=\int k_{F} \mathrm{~d} x$, where the Fermi wave number, $k_{F}$, is a function of the electrostatic potential, and the integral is taken over the electron's path.

We are looking for the phase difference, $\Delta \varphi$, of an electron travelling over two alternative paths in the two branches of the loop, at the point where both the paths recombine. This phase difference can be straight written as

$$
\Delta \varphi=\Delta k_{\mathrm{F}} L=\left[\frac{\mathrm{d} E(k)}{\mathrm{d} k} \frac{\mathrm{d} n(E)}{\mathrm{d} E}\right]^{-1} N
$$

where $E(k)$ is a dispersion relation between the electron energy $E$ and its wave number $k . \mathrm{d} n(E) / \mathrm{d} E$ stands here for the one-dimensional density of states, whose explicit form, including spin degeneracy, is [4]

$$
\frac{\mathrm{d} n(E)}{\mathrm{d} E}=\frac{2}{\pi}\left[\frac{\mathrm{d} E(k)}{\mathrm{d} k}\right]^{-1}
$$




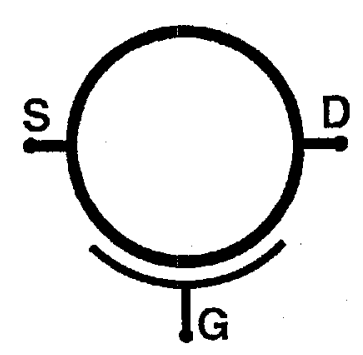

Fig. 1. Scheme of mesoscopic conducting loop where a gate electrode, $G$, is coupled capacitively to its lower branch.

and $N$ is the number of excess electrons induced in the wire by the gate potential. Inserting Eq. (2) into Eq. (1) one obtains an extremely simple result

$$
\Delta \varphi=\frac{\pi}{2} N \text {. }
$$

Surprisingly, $\Delta \varphi$ turns out to be independent of the electron energy, which is a particular feature of one-dimensional systems. Equation (3) exhibits a striking property of the considered system that a single elementary charge placed on the gate-wire capacitor leads to an essential phase difference: $\Delta \varphi=\pi / 2$. It means that the $\mathrm{eAB}$ effect belongs in fact to the category of single-electron phenomena, which carries significant consequences.

It causes, first of all, that the $\mathrm{AB}$ interference in a simple conducting loop will be inherently destroyed by charge fluctuations appearing on the gate-wire capacitor. Such fluctuations, well-known in the physics of single-electron phenomena in solids, are of either thermal or quantum origin. A detailed description of this finding will be published elsewhere [5].

We turn now attention to the possibility of the use of an open ballistic quantum dot instead of a mesoscopic quantum-wire loop to realise a controllable electrostatic Aharonov-Bohm interference. The essence of this possibility consists in that overall conductance through a regular (i.e. non-chaotic) dot may be dominated by only a few quasi-classical trajectories [6]. At a suitable low temperature, quantum interference between alternative paths reflected at the opposite lateral walls of the dot can be tuned by a difference in the bias voltage applied to lateral gate electrodes thus giving rise to the eAB-effect-controlled conductance.

We consider a square quantum dot defined by the split-gate technique on the GaAs/AlGaAs heterostructure containing two-dimensional electron gas. The potential profile in the dot can be varied by tuning the bias voltage applied to the lateral (plunger) Schottky gates, $G_{2}$ and $G_{2}^{\prime}$ (Fig. 2), and it will be further assumed to have a parabolic form [7]

$$
e \Phi=-E_{\mathrm{F}}^{0}\left(\frac{y}{w}\right)^{2}
$$

where $E_{\mathrm{F}}^{0}$ is the Fermi energy in the middle of the dot, and the co-ordinate axes and relevant dimensions are defined in Fig. 3.

The front and back gates of the dot are supplied with quantum point contact-like openings (Fig. 2). These openings are so adjusted that a few transverse 


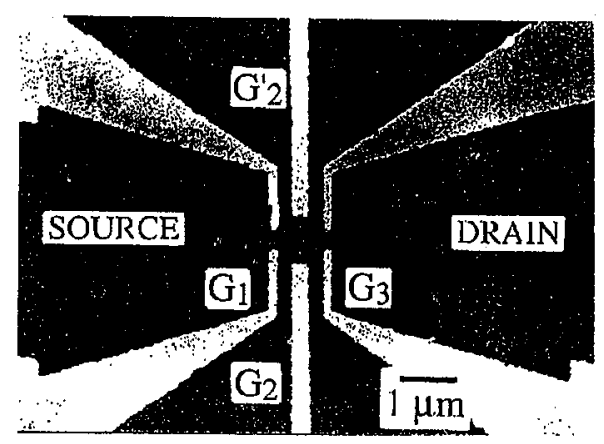

Fig. 2. Quantum dot defined by biased Schottky gates deposited on the surface of GaAs/AlGaAs heterostructure that depletes 2DEG at the interface beneath the gates. $G_{1}$ - entry gates, $G_{2}, G_{2}^{\prime}$ - lateral gates, $G_{3}$ - exit gates. Pattern obtained with electron-beam lithography and wet etching in co-operation with E. Kamińska and A. Piotrowska (Institute of Electron Technology, Warsaw) and J. Wróbel (Institute of Physics, Polish Academy of Sciences, Warsaw).

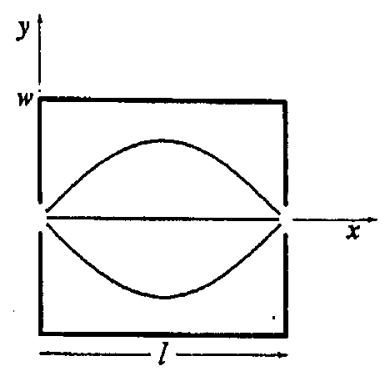

Fig. 3. Scheme of open quantum dot with parabolic potential profile where three simplest ballistic-electron trajectories are shown linking the entry and exit openings.

modes can propagate through them. Consequently, electrons are expected to enter the dot in a collimated beam which is diffracted to directions considerably away from the axis of the point contact. Such electrons will bounce back and forth between different walls describing in general complicated trajectories. From a variety of trajectories, we can distinguish three of the shortest lengths for which the quantum-phase coherence has the largest chance to survive. We expect that these trajectories will dominate coherent transport through the dot. The shortest trajectory is that linking directly the entry and exit openings. Electron transmission over this path can be diminished by suitable adjusting the bias voltage applied to the side gates [8]. The other two trajectories (lateral paths) link the two openings after single reflections at the opposite lateral walls of the dot (Fig. 3).

Classical trajectory of an electron at the Fermi energy which starts from the entry opening, is described by the following equation:

$$
y=w \sin \Theta \sin \left(\frac{x}{w \cos \Theta}\right),
$$


where $\Theta$ is the angle with respect to the $x$-axis at which the ballistic electron starts to travel through the dot. We are looking for the trajectories which terminate at the exit opening. For the two lateral trajectories being of the present interest, the angle $\Theta$ has to fulfil the following condition:

$$
\cos \Theta=\frac{l}{\pi w} \text {. }
$$

Then, an electron travelling from the entry to the exit acquires a phase

$$
\varphi=\frac{\pi}{4} k_{\mathrm{F}}^{0} w\left[1-\left(\frac{l}{\pi w}\right)^{2}\right],
$$

which is of course the same for both the upper and lower paths, where $k_{\mathrm{F}}^{0}$ is the Fermi wave number in the middle of the dot.

We introduce now an additional degree of freedom by differentiating the bias voltages applied to each of the lateral gates. It results in different potentials experienced by an electron in the upper and lower halves of the dot, which can be taken into account by putting into Eq. (4) $w+\Delta w$ and $w-\Delta w$ instead of $w$, respectively. The resulting phase difference of these two paths at the exit opening is now

$$
\Delta \varphi=\frac{\pi}{2} k_{\mathrm{F}}^{0} \Delta w\left[1+\left(\frac{l}{\pi w}\right)^{2}\right] .
$$

Although a relation between $\Delta w$ and the applied bias voltage is not easy to calculate, Eq. (8) clearly points out the possibility of an observation of the eAB effect in a dot while changing $\Delta w$. Relevant experiments are under performance.

This work was partly supported by the Committee for Scientific Research (Poland) under grant No. PBZ 28.11/P9.

\section{References}

[1] D. Takai, K. Ohta, Phys. Rev. B 49, 1844 (1994).

[2] S. Washburn, H. Schmid, D. Kern, R.A. Webb, Phys. Rev. Lett. 59, 1791 (1987).

[3] A. van Oudenaarden, M.H. Devoret, Yu.V. Nazarov, J.E. Mooij, Nature 391, 768 (1998).

[4] C.W.J. Beenacker, H. van Houten, Solid State Phys. 44, 1 (1991).

[5] T. Figielski, T. Wosiński, submitted to Appl. Phys. Lett.

[6] D.K. Ferry, R. Akis, J.P. Bird, Superlattices Microstruct. 23, 611 (1998).

[7] S.E. Laux, D.J. Frank, F. Stern, Surf. Sci. 196, 101 (1998).

[8] R. Schuster, K. Ensslin, in: Proc. 23rd Int. Conf. Phys. Semiconductors, Eds. M. Scheffler, R. Zimmermann, World Scientific, Singapore 1997, p. 1557. 